\title{
The expression of translocator protein in human thyroid cancer and its role in the response of thyroid cancer cells to oxidative stress
}

\author{
Joanna Klubo-Gwiezdzinska, ${ }^{1,2}$, Kirk Jensen ${ }^{3}$, Andrew Bauer ${ }^{3}$, Aneeta Patel ${ }^{3}$, John Costello Jr ${ }^{3}$, \\ Kenneth D Burman ${ }^{1}$, Leonard Wartofsky ${ }^{1}$, Matthew J Hardwick ${ }^{4}$ and Vasyl V Vasko ${ }^{3}$ \\ ${ }^{1}$ Division of Endocrinology, Department of Medicine, Washington Hospital Center, 110 Irving Street Northwest, Washington, \\ District of Columbia 20010-2910, USA \\ ${ }^{2}$ Department of Endocrinology and Diabetology, Collegium Medicum in Bydgoszcz, Nicolaus Copernicus University in Torun, ul. M. Sklodowskiej- Curie 9, \\ 85-094 Bydgoszcz, Poland \\ ${ }^{3}$ Department of Pediatrics, Uniformed Services University of the Health Sciences, 4301 Jones Bridge Road, Bethesda, Maryland 20814-4712, USA \\ ${ }^{4}$ Laboratory of Clinical Investigations, Medstar Research Institute, Washington Hospital Center, 110 Irving Street Northwest, Washington, \\ District of Columbia 20010-2910, USA \\ (Correspondence should be addressed to V V Vasko; Email: vvasko@usuhs.edu)
}

\begin{abstract}
The translocator protein (TSPO), formerly known as a peripheral benzodiazepine receptor, exerts pro-apoptotic function via regulation of mitochondrial membrane potential. We examined TSPO expression in human thyroid tumors (25 follicular adenomas (FA), 15 follicular cancers (FC), and 70 papillary cancers (PC)). The role of TSPO in the regulation of cell growth, migration, and apoptosis was examined in thyroid cancer cell lines after TSPO knockdown with siRNA and after treatment with TSPO antagonist (PK11195). Compared with normal thyroid, the level of TSPO expression was increased in FA, FC, and PC in 24, $26 \cdot 6$, and $55 \cdot 7 \%$ of cases respectively. Thyroid cancer cell lines demonstrated variable levels of TSPO expression, without specific association with thyroid oncogene mutations. Treatment with inhibitors of PI3K/AKT or MEK/ERK signaling was not associated with changes in TSPO
\end{abstract}

expression. Treatment with histone deacetylase inhibitor (valproic acid) increased TSPO expression in TSPO-deficient cell lines (FTC236 cells). TSPO gene silencing or treatment with PK11195 did not affect thyroid cancer cell growth and migration but prevented depolarization of mitochondrial membranes induced by oxidative stress. Induction of TSPO expression by valproic acid was associated with increased sensitivity of FTC236 to oxidative stress-inducible apoptosis. Overall, we showed that TSPO expression is frequently increased in PC. In vitro data suggested the role of epigenetic mechanism(s) in the regulation of TSPO in thyroid cells. Implication of TSPO in the thyroid cancer cell response to oxidative stress suggested its potential role in the regulation of thyroid cancer cell response to treatment with radioiodine and warrants further investigation.

Journal of Endocrinology (2012) 214, 207-216

\section{Introduction}

Thyroid cancer is the most common endocrine malignancy. Patients with distant metastases have a poor prognosis with a reported 10-year survival rate after detection of distant metastases ranging from 25 to $42 \%$ (Schlumberger \& Sherman 2009). Genetic alterations in antiapoptotic signaling pathways are implicated in thyroid cancer cell survival in metastatic sites (Liu et al. 2008), but the exact mechanisms that underlie resistance to apoptosis in thyroid cancer cells are not completely elucidated.

Mitochondria-mediated apoptosis plays an important role in tissue homeostasis, and its alteration is associated with cancer. The translocator protein (TSPO; $18 \mathrm{kDa}$ ), previously known as the peripheral benzodiazepine receptor, is a mitochondrial protein involved in the regulation of mitochondrial membrane potential (Chelli et al. 2004) and mitochondria-mediated apoptosis (Papadopoulos et al. 2006, Veenman et al. 2008).

Previous studies demonstrated that apoptotic action of free radical and irradiation-generated reactive oxygen species are associated with mitochondrial membrane depolarization (Satoh et al. 1997). It has also been shown that free radicals are implicated in the topographical reorganization of TSPO, resulting in TSPO association with voltage-dependent anion channels (VDACs) and adenine nucleotide translocator (ANT). TSPO interaction with VDACs and ANT leads to mitochondrial transition pore opening, VDAC-mediated release of cytochrome $c$ into the cytosol, and activation of the mitochondrial apoptosis pathway (Veenman et al. 2008).

TSPO is ubiquitously expressed throughout the body and is particularly enriched in steroidogenic tissues including 
ovary, testis, adrenal, and placenta (Corsi et al. 2008). Aberrant TSPO expression has been shown in different malignancies. High levels of TSPO were detected in glioblastomas, prostate, breast, and colon cancers, while decreased levels of TSPO were found in squamous cell lung carcinoma and anaplastic astrocytoma (Hardwick et al. 1999, Maaser et al. 2005, Takaya et al. 2007, Vlodavsky \& Soustiel 2007, Fafalios et al. 2009). The role of TSPO in cancer progression was suggested previously, as increased levels of TSPO were observed in metastases compared with primary cancers (Fafalios et al. 2009).

The functional role of TSPO in cancer cells was largely explored using TSPO-specific ligands such as the isoquinoline carboxamide PK11195. At nanomolar concentrations, PK11195 inhibits TSPO-mediated opening of mitochondrial transition pores and prevents apoptosis (Kugler et al. 2008, Shoukrun et al. 2008, Zeno et al. 2009). At micromolar concentrations, however, TSPO ligands induced apoptosis in cell lines derived from breast, esophageal, colon, and prostate cancers (Maaser et al. 2001, Sutter et al. 2004, Akech et al. 2005).

Despite the important role of TSPO in the regulation of mitochondria-mediated apoptosis, there are no reports on TSPO expression in thyroid cancer. In this study, we analyzed the patterns of TSPO expression in human thyroid tumors and examined TSPO functions using thyroid cancer cell lines.

\section{Materials and Methods}

\section{Human thyroid tissue samples}

The protocol for the study was approved by the Institutional Review Board at the Washington Hospital Center and Uniformed Services University of the Health Sciences. Thyroid tissue samples were paraffin embedded and histological diagnoses were established according to the WHO classification. There were 25 follicular adenomas (FAs), 15 follicular cancers (FCs), and 70 papillary cancers (PCs). We also examined lymph node metastases (LNMs) removed at the time of surgery from 22 patients.

\section{Immunohistochemical analysis of human biopsies}

Immunostaining was performed on paraffin-embedded tissue sections. Endogenous peroxidase activity was quenched by incubation in $3 \% \mathrm{H}_{2} \mathrm{O}_{2}$. Sections were incubated overnight with anti-TSPO polyclonal antibody (Trevigen, Gaithersburg, MD, USA) and immunostaining was performed using Vector kits (Vector Labs, Burlingame, CA, USA) according to the manufacturer's instruction. Negative controls were performed as earlier except that the primary antisera were not included. The results of staining were characterized as follows: 0 - no staining, 1 - focal/low intensity staining in $<10 \%$ of cells, 2 - strong staining in up to $50 \%$ of cells, 3 - strong staining in more than $50 \%$ of cells.
The immunoactivity was scored independently by two investigators (V V V and J K-G) on a 0-3 scale. Scoring was compared and averages were generated.

\section{Cell cultures and reagents}

Human thyroid cancer cell lines derived from follicular thyroid cancer (FTC133, FTC236, and FTC238) and papillary thyroid cancer (TPC1, KTC, and BCPAP) were obtained from Dr Motoyasu Saji (The Ohio State University) with permission from the researchers who originally established the cell lines. We also used HEK293 cells for overexpression of thyroid oncogenes. All thyroid cancer cell lines had been tested and authenticated by DNA analysis to be of thyroid origin. Cancer cells were propagated in RPMI 1640 medium (Invitrogen) supplemented with 5\% fetal bovine serum. To evaluate the effects of the TSPO-specific antagonist, cells were incubated with either control medium or medium containing PK11195 (Sigma Chemical Co.). The pharmacological inhibitor of PI3K/AKT signaling (LY-294002) was from Sigma Chemical Co., and the MEK1/2 inhibitor (U-0126) was from Cell Signaling (Danvers, MA, USA).

\section{Cells transfections}

FTC133, TPC1, and BCPAP cells were transfected with scrambled control siRNA or TSPO-specific siRNA (Dharmacon, Lafayette, CO, USA) using Lipofectamine RNAiMAX transfection reagent (Life Technologies). The cells were incubated with the liposome complexes for $24 \mathrm{~h}$. Assays were performed $48 \mathrm{~h}$ after the beginning of the transfections.

HEK293 cells were transiently transfected with RET/PTC1 or RET/PTC3 cDNA obtained from Dr Motoyasu Saji (The Ohio State University) or control vector using Lipofectamine 2000 (Life Technologies) according to the manufacturer's protocol. Immunostaining with anti-RET and anti-TSPO antibodies was performed $48 \mathrm{~h}$ after transfection.

\section{RNA extraction and determination of TSPO $m R N A$ using real-time PCR}

Total RNA was isolated from thyroid cancer cells using TRIzol reagent (Invitrogen) according to the manufacturer's protocol. Quantitative real-time PCR was performed using the following primers: TSPO - forward GGG CAC GCT CTA CTC AGC, TSPO - reverse GGA ACC ACA GCC TTC TCT GT. SYBR green-based quantitative PCR master mixes were from SuperArray Bioscience Corporation (Frederick, MD, USA).

\section{Protein extraction and western blot analysis}

Thyroid cancer cells were incubated with ice-cold cell lysis buffer, scraped, and centrifuged and the supernatant was 
stored at $-80{ }^{\circ} \mathrm{C}$. Total protein lysate $(25 \mu \mathrm{g})$ was suspended in reduced SDS sample buffer and protein lysates were subjected to SDS-PAGE (7\%). The separated proteins were transferred to nitrocellulose membranes $(0 \cdot 2 \mu \mathrm{m}$ pore size; Invitrogen) by electrophoretic blotting (Invitrogen).

Membranes were incubated overnight with the primary anti-TSPO antibodies, p-Akt1/2/3 (Ser472, SC-7985), pERK (SC-7388), and cleaved caspase-3 antibody. Immunodetection of proteins was performed using Li-Cor Odyssey imaging system (LI-COR Biosciences, Lincoln, NE, USA).

\section{Immunofluorescent staining of thyroid cancer cells}

Thyroid cancer cells were cultured overnight on eightchambered SuperCell Culture Slides (Fisher Scientific, Pittsburgh, PA, USA) and then fixed in formalin for $15 \mathrm{~min}$. After washing, cells were incubated overnight at $4{ }^{\circ} \mathrm{C}$ with anti-TSPO, anticleaved caspase-3, and anti-RET antibodies. Slides were then incubated at room temperature for $1 \mathrm{~h}$ with species-specific Alexa 488 conjugated secondary antibody.

\section{Detection of mitochondrial membrane potential}

Cells were plated in 96-well plates. Mitochondrial membrane potential was assessed using the fluorogenic lipophilic cation (JC-1) (Cayman Chemical Company, Ann Arbor, MI, USA). In cells with hyperpolarized mitochondrial membranes, JC-1 spontaneously forms complexes (J-aggregates) with intense red fluorescence. In cells with depolarized mitochondrial membranes, JC-1 remains in the monomeric forms, which do not show red fluorescence. Detection of mitochondrial membrane potential was performed according to the manufacturer's instructions followed by fluorescent microscopy.

\section{Migration assay}

Cancer cells were grown until 30-40\% confluent, washed with PBS, trypsinized, and centrifuged for $5 \mathrm{~min}$. A volume of 30000 cells in $300 \mu \mathrm{l} 0 \cdot 2 \%$ FBS medium was placed into the upper chamber of Boyden Chamber $(8 \mu \mathrm{m}$ pore $)$ inserts in 24-well plates filled with $400 \mu$ RPMI containing $10 \% \mathrm{FBS}$ in the bottom chamber. Cells were incubated at $37^{\circ} \mathrm{C}$ in $5 \% \mathrm{CO}_{2}$ for $24 \mathrm{~h}$. The cells on the Boyden Chamber membrane were stained using Diff-Quick protocol (IMEB, Inc., San Marcos, CA, USA).

\section{Cell proliferation and detection of cell viability}

Thyroid cancer cells were plated at a density of 50000 cells/well in six-well plates. Cells adhered overnight and then were treated with PK11195 at concentrations varying from $10 \mathrm{nM}$ to $100 \mu \mathrm{M}$. After incubation for 24,48 , or $72 \mathrm{~h}$, the cell proliferation rate was determined by cell counting using the
Vi-CELL Cell Viability Analyzer from Beckman Coulter (Fullerton, CA, USA).

For induction of oxidative stress, thyroid cancer cells were treated with $\mathrm{H}_{2} \mathrm{O}_{2} . \mathrm{H}_{2} \mathrm{O}_{2}$ stock solution $(100 \mathrm{mM})$ was added into cell culture medium to achieve the desired working concentration. For TSPO ligand studies, PK11195 was added to the culture medium together with $\mathrm{H}_{2} \mathrm{O}_{2}$.

Cell viability was determined by Alamar Blue assay and dead cells were detected using propidium iodine staining. All experiments were repeated at least three times, and the average values \pm s.D. of representative experiments are reported.

\section{Results}

TSPO immunodetection in human thyroid tissue samples

Fifty-six normal thyroid samples were evaluated for their pattern of TSPO expression by immunohistochemistry using a TSPO-specific antibody. No evidence of TSPO expression was detected in 46 of 56 normal thyroid tissue samples. In the remaining cases, TSPO immunoreactivity was associated with the presence of lymphocyte infiltration.

The results of TSPO immunodetection in thyroid tumors are summarized in Table 1 . Six of 25 examined FAs (24\%) demonstrated high levels of TSPO expression (score 2 and 3). Compared with normal thyroid tissue, the level of TSPO expression was significantly higher in FAs $(P=0 \cdot 001)$. The intensity of TSPO immunostaining in FAs correlated with the presence of cellular atypia. Among six cases with high levels of TSPO expression, there were two Hurthle cell adenomas and four adenomas with limited nuclear features of thyroid cancer (Fig. 1). Eighty-five thyroid cancers (15 FCs and 70 PCs) were examined for TSPO expression by immunostaining. Compared with the normal thyroid tissue, the level of TSPO expression was increased in 43/85 (50.5\%) of examined tumors $(P=0 \cdot 0001)$. In FCs and PCs, the high level of TSPO staining was detected in 4/15 (26.6\%) and in $39 / 70(55 \cdot 7 \%)$ of cases respectively. Among PCs, cytoplasmic TSPO immunoreactivity was more frequently found in PCs with typical papillary morphology $(37 / 56 ; 66 \cdot 0 \%)$ compared with follicular variant of PCs (FVPCs) $(2 / 14 ; 14 \cdot 2 \%)$.

Table 1 Results of TSPO immunostaining in human thyroid tissue samples

\section{TSPO immunostaining score}

\begin{tabular}{|c|c|c|c|c|c|}
\hline & & & & & \\
\hline Histology & No. of cases & $\overline{0}$ & 1 & 2 & 3 \\
\hline Normal thyroid & 56 & 46 & 6 & 4 & 0 \\
\hline FA & 25 & 8 & 7 & 6 & 0 \\
\hline FC & 15 & 7 & 4 & 2 & 2 \\
\hline FVPC & 14 & 2 & 10 & 1 & 1 \\
\hline PC & 56 & 9 & 10 & 21 & 16 \\
\hline LNM & 22 & 2 & 3 & 12 & 5 \\
\hline
\end{tabular}



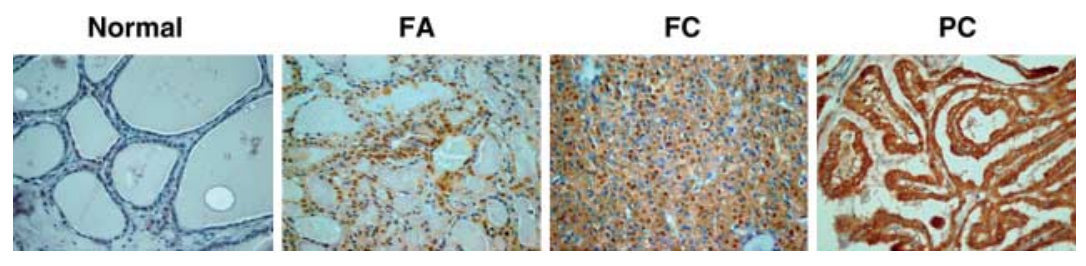

Figure 1 TSPO expression in normal thyroid, FA, FC, and PC.

The intensity of TSPO immunostaining was associated with tumor histological types and was significantly higher in thyroid cancer with papillary morphology compared with thyroid cancer with follicular patterns of growth $(P=0 \cdot 003)$. In LNMs that were removed at the time of initial surgery, the patterns of TSPO staining were similar to those observed in primary tumors.

\section{Regulation of TSPO expression in thyroid cancer cell lines}

TSPO mRNA was detected in all examined thyroid cancer cells lines as well as in commercially available RNA (Stratagene, Santa Clara, CA, USA) from normal human thyroid. Consistent with human data, increased mRNA of TSPO was detected in all PC-derived cell lines compared with normal thyroid. The TSPO mRNA level in TPC1, BCPAP, KTC, and FTC133 were significantly higher compared with the level observed in the normal thyroid (Fig. 2A). In BCPAP cells, TSPO mRNA was significantly lower than in TPC1, KTC, and FTC133 cells. However, there were no statistically significant differences in TSPO mRNA between TPC1, KTC, and FTC133 cells. Only FTC236 and FTC238 cells demonstrated significantly lower levels of TSPO compared with normal thyroid and other cell lines. Interestingly, FTC236 and FTC238 cells, derived from recurrent lesions, demonstrated lower levels of TSPO compared with FTC133 cells, derived from primary lesions of the same patient.

Analysis of TSPO protein by western blot revealed expression of TSPO polymer $(36 \mathrm{kDa})$ in all examined thyroid cancer cells (Fig. 2B). The expression of TSPO monomer $(18 \mathrm{kDa})$ was detected in TPC1, BCPAP, KTC, and FTC133 cells but not in FTC236 and FTC238 cells. Immunostaining demonstrated that TSPO was distributed within the cytoplasm of thyroid cancer cells (Fig. 2C).

As human thyroid cancers and cell lines demonstrated high levels of TSPO expression, we sought to determine the possible role of thyroid oncogene-inducible signaling in the regulation of TSPO and examined the effects of $\mathrm{PI} 3 \mathrm{~K} / \mathrm{AKT}$ and MAPK/ERK inhibitors on TSPO expression. At baseline conditions, the highest level of AKT activation was detected in PTEN-deficient thyroid cancer cell lines (FTC133, FTC236, and FTC238). Thyroid cancer cells harboring a BRAF mutation (BCPAP and KTC) demonstrated high levels of ERK activation, and RET/PTC1-positive TPC1 cells were characterized by constitutive activation of both
pAKT and pERK. Treatment with PI3K/AKT inhibitor (LY294002) or MEK inhibitor (U026) for 24 and $48 \mathrm{~h}$ resulted in inhibition of $\mathrm{pAKT}$ and $\mathrm{pERK}$, respectively, but did not significantly affect TSPO mRNA (data not shown) or protein levels (Fig. 3A and B) in any of the examined cell lines. To confirm these findings, we examined TSPO expression in HEK293 cells transfected with RET/PTC1 and RET/PTC3. Forced expression of RET/PTC1 or RET/PTC3 did not induce TSPO expression in HEK293 cells (Fig. 3C).

Recent data suggested the role of epigenetic mechanisms in the regulation of TSPO expression in breast cancer cell lines (Batarseh et al. 2012). Therefore, we next examined whether

A

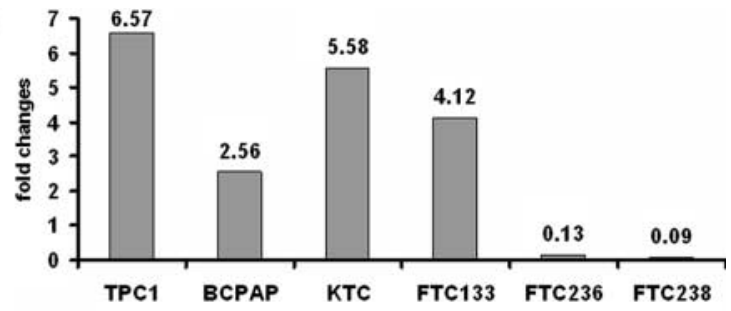

B

B TPC BCP KTC 133236238

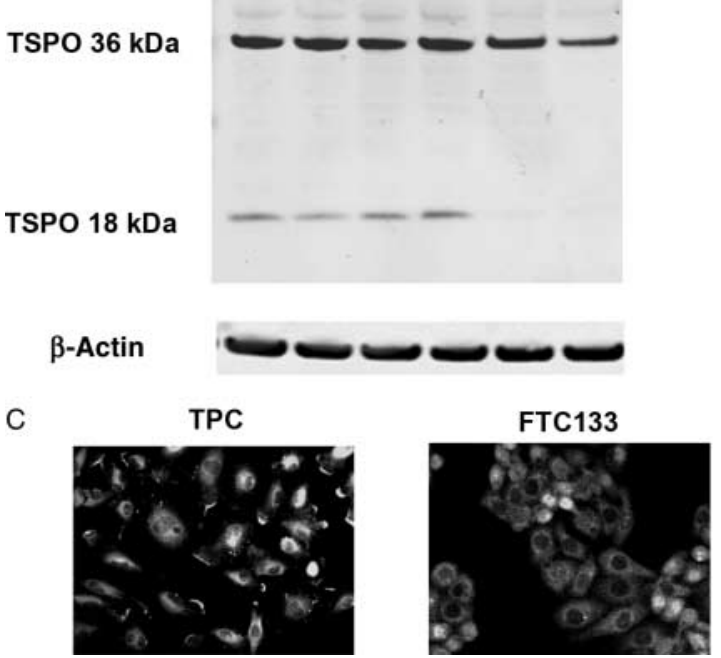

Figure 2 TSPO expression in human thyroid cancer cell lines. (A) The relative level of TSPO mRNA in thyroid cancer cell lines compared with the mRNA from normal thyroid cells. (B) TSPO protein levels documented by western blot. (C) Immunostaining showing cytoplasmic localization of TSPO. 

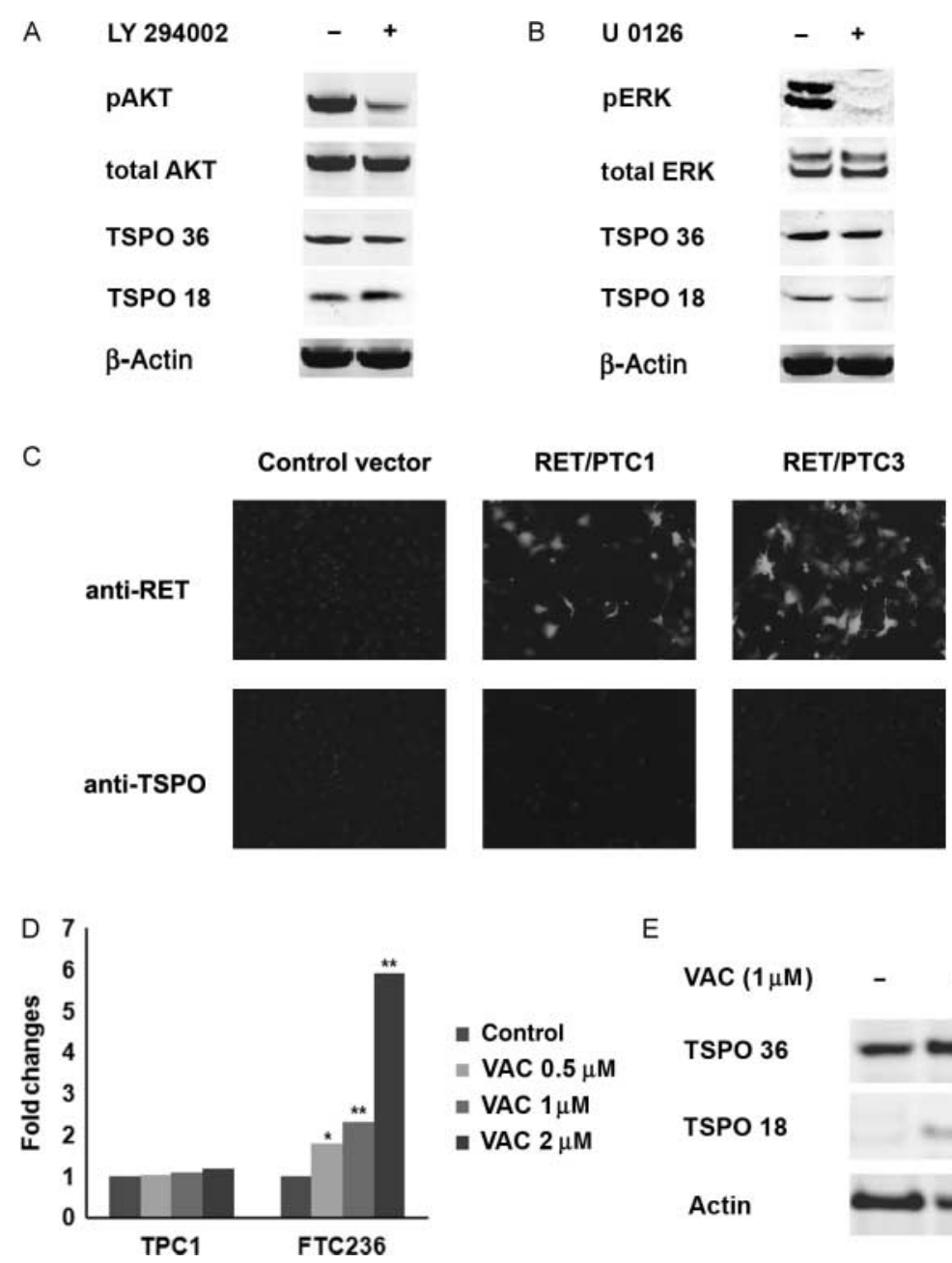

E

Figure 3 The effects of thyroid cancer oncogene-induced signaling and epigenetic mechanisms on TSPO expression. (A) Treatment with PI3K/AKT inhibitor LY294002 resulted in downregulation of pAKT but had no effect on TSPO expression in TPC1 cells. (B) Treatment with MEK inhibitor (U026) resulted in downregulation of $p E R K$ but no effect on TSPO expression in TPC1 cells. (C) Immunostaining with anti-RET and anti-TSPO showing that forced expression of RET/PTC1 or RET/PTC3 did not induce TSPO expression in HEK293 cells. (D) Graph demonstrating relative TSPO mRNA expression in thyroid cells treated with valproic acid (VAC). VAC had no effects on TSPO mRNA expression in TPC1 cells with intrinsically high level of TSPO but induced TSPO mRNA expression in FTC236 cells characterized by low baseline TSPO level. * $<0 \cdot 05$ and ${ }^{* *}<0 \cdot 001$. (E) Induction of TSPO protein expression in FTC236 cells treated with valproic acid.

TSPO expression in thyroid cancer cell lines could be affected by treatment with the histone deacetylase inhibitor valproic acid. Treatment with valproic acid at concentrations $1,2 \cdot 5$, and $5 \mathrm{mM}$ had no effects on TSPO mRNA expression in cell lines with an intrinsically high level of TSPO but did induce TSPO mRNA expression in FTC236 cells (Fig. 3D). Quantification of results showed that the TSPO mRNA level was increased in FTC236 cells after treatment with valproic acid at concentration 1 and $2 \mathrm{mM}$ by $2 \cdot 3$-fold $(P=0 \cdot 004)$ and $5 \cdot 94$-fold $(P=0 \cdot 001)$ respectively. Induction of TSPO expression in FTC236 cells after treatment with valproic acid was confirmed by western blot analysis (Fig. 3E).
Together, these data show that differential expression of TSPO in thyroid cancer cell lines appears to be independent of thyroid oncogene mutations and activation of PI3K/AKT or MEK/ERK signaling. Induction of TSPO by treatment with valproic acid suggests the role of epigenetic mechanisms in the regulation of TSPO in thyroid cancer cells.

The role of TSPO in the regulation of thyroid cancer cell growth and motility

To determine the functional role of TSPO, we first examined the role of TSPO silencing on thyroid cancer cell growth, 
Table 2 Results of TSPO silencing in thyroid cancer cell lines

\begin{tabular}{|c|c|c|c|c|}
\hline Cell lines & Scramble $\left(C_{t}\right.$ value $)$ & siTSPO $\left(C_{t}\right.$ value $)$ & Fold change & $P$ value \\
\hline \multicolumn{5}{|l|}{ FTC133 } \\
\hline TSPO & $22 \cdot 36 \pm 0 \cdot 35$ & $29 \cdot 81 \pm 0 \cdot 35$ & \multirow[t]{2}{*}{$-138 \cdot 62$} & \multirow[t]{2}{*}{$0 \cdot 0022$} \\
\hline $18 \mathrm{~s}$ & $7.93 \pm 0.06$ & $8 \cdot 25 \pm 0.02$ & & \\
\hline \multicolumn{5}{|l|}{ TPC1 } \\
\hline TSPO & $20 \cdot 48 \pm 0 \cdot 08$ & $25 \cdot 94 \pm 0 \cdot 11$ & \multirow[t]{2}{*}{$-36 \cdot 5$} & \multirow[t]{2}{*}{$0 \cdot 0003$} \\
\hline $18 \mathrm{~s}$ & $7 \cdot 19 \pm 0 \cdot 11$ & $7 \cdot 46 \pm 0 \cdot 11$ & & \\
\hline \multicolumn{5}{|l|}{ BCPAP } \\
\hline TSPO & $23 \cdot 66 \pm 0 \cdot 1$ & $28 \cdot 77 \pm 0 \cdot 02$ & \multirow[t]{2}{*}{$-39 \cdot 88$} & \multirow[t]{2}{*}{$0 \cdot 0002$} \\
\hline $18 \mathrm{~s}$ & $8 \cdot 16 \pm 0 \cdot 13$ & $7 \cdot 8 \pm 0 \cdot 06$ & & \\
\hline
\end{tabular}

using TSPO-specific siRNA. Forty-eight hours following transfection, cells were harvested and RNA was subjected to RT-PCR. The efficiency of silencing was confirmed by analysis of TSPO mRNA (Table 2) and protein levels (Fig. 4A and B). The rate of growth was determined by direct cell count in FTC133 and TPC1 cells transfected with scramble and TSPO-specific siRNA at 48 and $72 \mathrm{~h}$ post-transfection. Tspo knockdown was not associated with inhibition of cell growth or cell viability. Cyclin D1 mRNA level was not significantly affected by TSPO silencing in FTC133 or TPC1 cells (data not shown).

We also examined the effect of the TSPO antagonist PK11195 on thyroid cancer cell growth and viability. Treatment with PK11195 (ranging from $10 \mathrm{nM}$ to $1 \mu \mathrm{M}$ ) had no effect on cell growth in any of the cancer cell lines (Fig. 4C). Exposure to PK11195 at concentrations ranging from 10 to $50 \mu \mathrm{M}$ inhibited thyroid cancer cell proliferation. At these concentrations, however, PK11195 induced cell death in thyroid cancer cells with intrinsically low levels of TSPO (FTC236 and FTC238), as well as in cells transfected with TSPO siRNA (data not shown), therefore suggesting potential nonspecific off-target effects.

Evaluation of thyroid cancer cell motility in the Boyden chamber assay showed no significant differences in migratory abilities in TSPO-rich (TPC1, BCPAP, KTC, and FTC133) and TSPO-deficient (FTC236 and FTC238) cells. TSPO silencing in cells with intrinsically high levels of TSPO (FTC133 and TPC1 cells) did not affect their migratory ability (Fig. 4D). Together, these data suggested that the level of TSPO expression is not directly associated with the rate of growth or migratory ability of thyroid cancer cells.

\section{Inhibition of TSPO prevents mitochondrial membrane depolarization in an oxidative stress model}

TSPO plays a role in the regulation of mitochondrial transition pore opening, which is critical for the execution of oxidative stress-inducible apoptosis. Therefore, we examined whether inhibition of TSPO would affect thyroid cancer cell response to oxidative stress.

FTC133 and TPC1 cells transfected with scramble and TSPO-specific siRNA were treated with increasing concentrations of $\mathrm{H}_{2} \mathrm{O}_{2}$. In FTC133 and TPC1 cells that were transfected with scramble vector, treatment with $\mathrm{H}_{2} \mathrm{O}_{2}$ was associated with loss of mitochondrial membrane potential as demonstrated by JC-1 staining (Fig. 5A). It has to be noted that despite similar level of TSPO expression in FTC133 and TPC1 cells, the sensitivity to $\mathrm{H}_{2} \mathrm{O}_{2}$ was different. Mitochondrial membrane depolarization was observed in FTC133
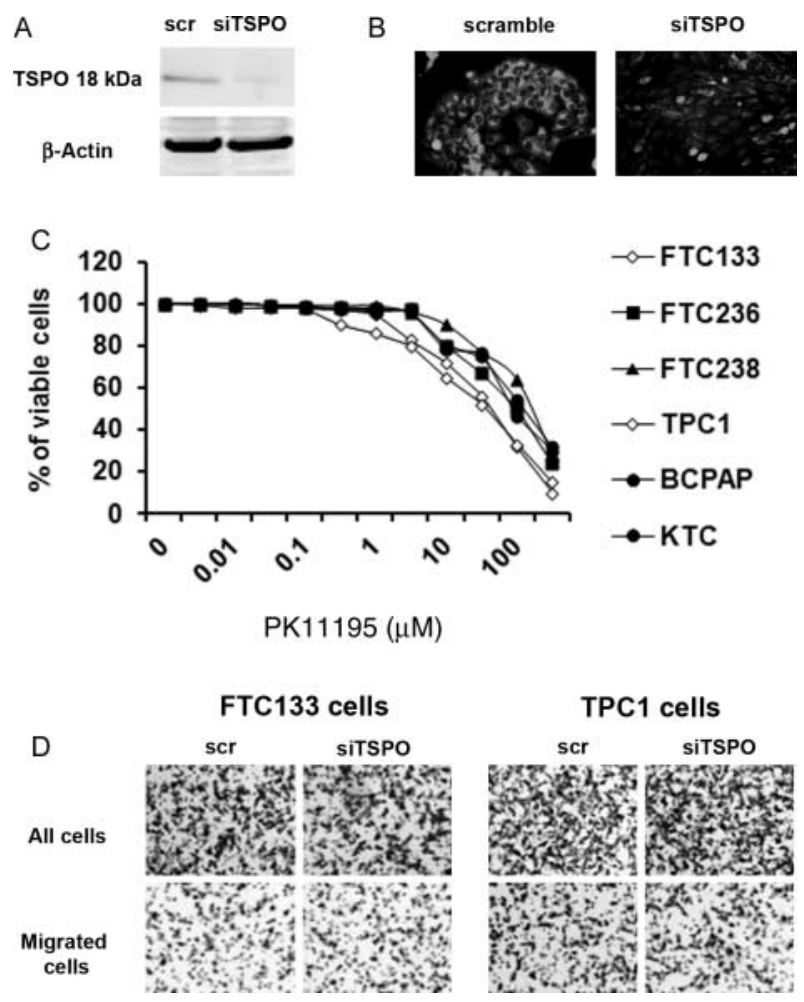

Figure 4 The effect of TSPO inhibition on thyroid cancer cell growth. (A) Western blot analysis documenting decreased levels of TSPO after cell transfection with TSPO-specific siRNA.

(B) Immunostaining showing loss of TSPO in TPC1 cells transfected with TSPO-specific siRNA. (C) Treatment with TSPO inhibitor PK11195 in concentrations ranging from $10 \mathrm{nM}$ to $1 \mu \mathrm{M}$ had no effect on cell growth in any of the cancer cell lines examined. Exposure to higher concentrations ranging from 10 to $50 \mu \mathrm{M}$ inhibited thyroid cancer cell growth. (D) Loss of TSPO expression did not affect thyroid cancer cell migration in Boyden chamber migration assay. 
A

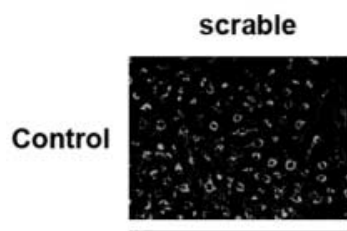

FTC133

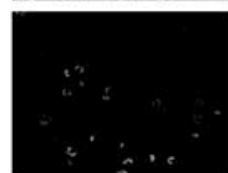

$50 \mu \mathrm{M}$
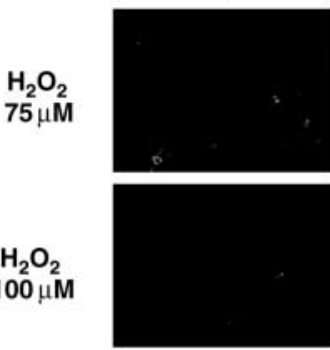

$\mathrm{H}_{2} \mathrm{O}_{2}$ $100 \mu \mathrm{M}$

B
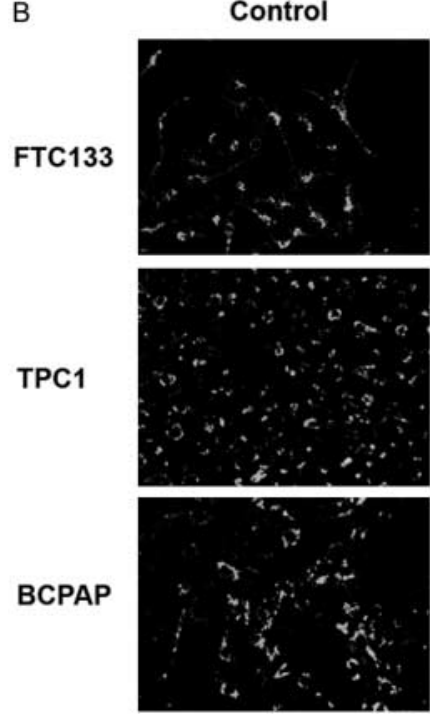

Figure 5 The effects of TSPO inhibition on thyroid cell response to oxidative stress.

(A) TSPO silencing attenuated $\mathrm{H}_{2} \mathrm{O}_{2}$-induced mitochondrial membrane depolarization.

(B) Thyroid cancer cell treatment with TSPO antagonist PK11195 (100 nM) attenuated

$\mathrm{H}_{2} \mathrm{O}_{2}$-inducible depolarization of mitochondrial membrane.
TPC1

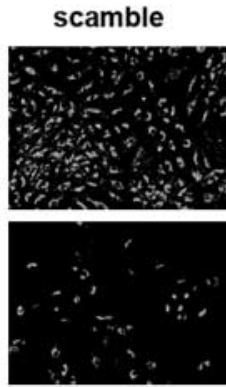

siTSPO
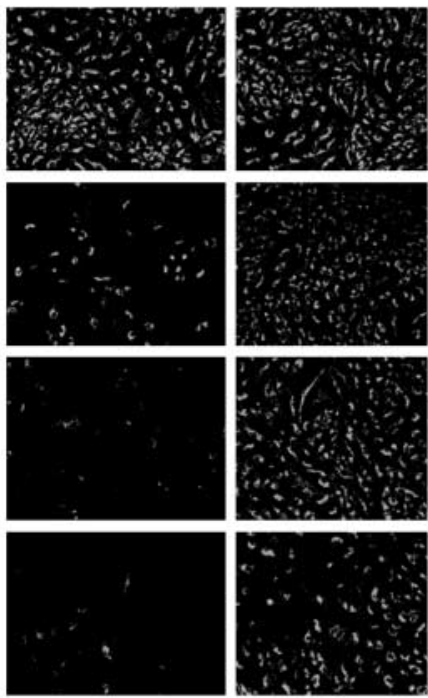

PK11195 \& $\mathrm{H}_{2} \mathrm{O}_{2}$
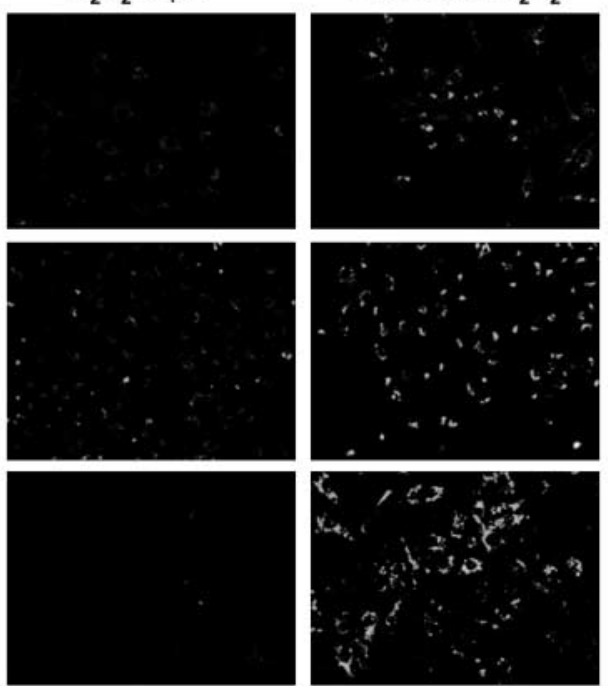

cells at a $\mathrm{H}_{2} \mathrm{O}_{2}$ concentration of $50 \mu \mathrm{M}$, and in TPC1 cells

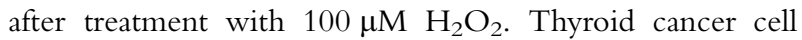
transfection with TSPO siRNA attenuated $\mathrm{H}_{2} \mathrm{O}_{2}$-inducible depolarization of mitochondrial membranes as demonstrated by JC-1 staining. We also examined whether TSPO antagonist PK11195 would have a protective effect in the oxidative stress model. Treatment of thyroid cancer cells expressing high levels of TSPO with PK11195 (100 nM) prevented oxidative stress-inducible depolarization of mitochondrial membrane (Fig. 5B).
Treatment with valproic acid sensitizes thyroid cancer cells to oxidative stress-inducible apoptosis

Finally, we examined whether induction of TSPO expression in TSPO-deficient thyroid cancer cells by treatment with valproic acid could increase their sensitivity to oxidative stress. Consistent with previously reported data (Shen et al. 2005), treatment with increasing concentrations of valproic acid (from 0.5 to $5 \mathrm{mM}$ ) resulted in dose- and time-dependent inhibition of thyroid cancer cell growth. In TSPO-deficient 

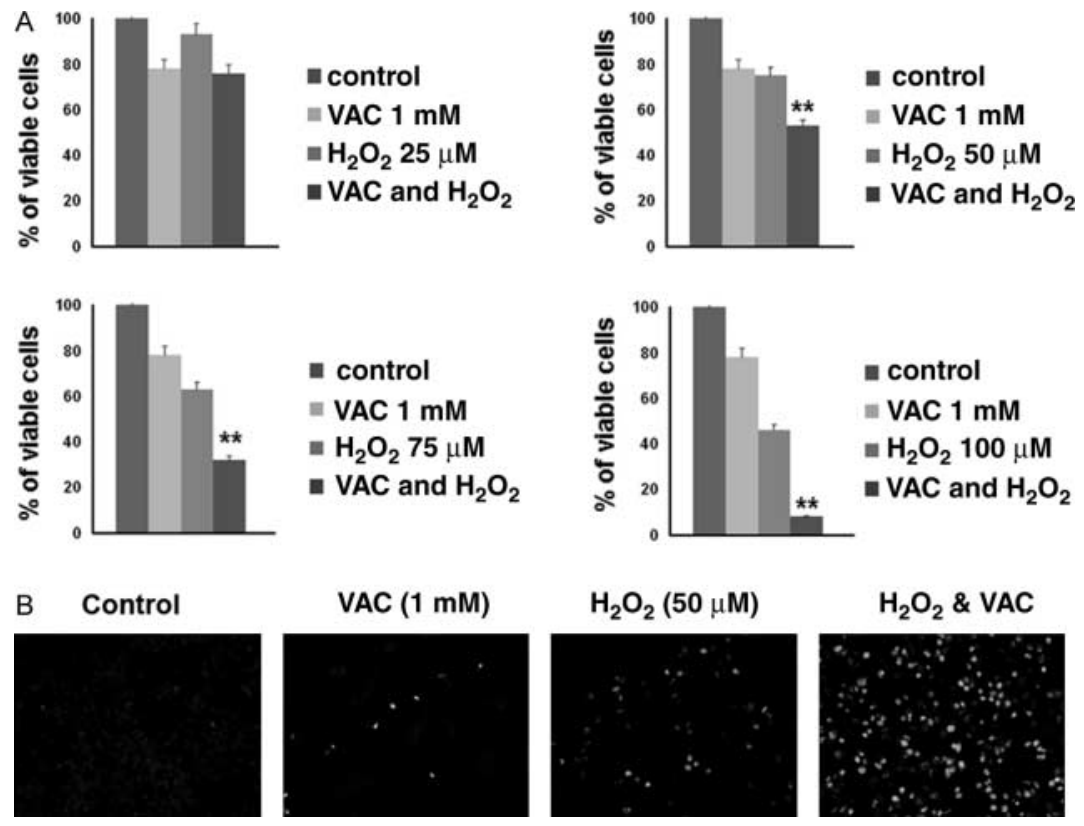

Figure 6 Treatment with valproic acid sensitizes thyroid cancer cells to oxidative stress. (A) Graphs showing the effects of valproic acid alone (VAC), increasing concentrations of $\mathrm{H}_{2} \mathrm{O}_{2}$ alone, and combination of VAC and $\mathrm{H}_{2} \mathrm{O}_{2}$ on viability of FTC236 cells. Valproic acid potentiates the effects of $\mathrm{H}_{2} \mathrm{O}_{2}$ on thyroid cancer cells. (B) Immunostaining with cleaved caspase- 3 indicates induction of apoptosis in FTC236 cells treated with combination of VAC with $\mathrm{H}_{2} \mathrm{O}_{2}$.

FTC236 cells, treatment with $1 \mathrm{mM}$ valproic acid (concentration sufficient for induction of TSPO expression) for $24 \mathrm{~h}$ decreased number of viable cells by $12 \%$. As demonstrated in Fig. 6A, pre-treatment of FTC236 cells with valproic acid $(1 \mathrm{mM})$ significantly potentiated the effects of $\mathrm{H}_{2} \mathrm{O}_{2}$. To determine whether the decreased number of viable cells was associated with induction of apoptosis, we examined caspase-3 cleavage. Immunostaining with antibodies against cleaved caspase-3 indicated that treatment with valproic acid sensitized FTC236 cells to oxidative stress-inducible apoptosis (Fig. 6B).

\section{Discussion}

In this study, we characterized TSPO expression in a panel of benign and malignant thyroid lesions and explored the functional role of TSPO using thyroid cancer cell lines. We have shown that TSPO overexpression is an early event during thyroid tumorigenesis, as FAs expressed significantly higher levels of TSPO compared with normal thyroid tissue. Analysis of malignant thyroid lesions revealed an association between tumor phenotypes and the level of TSPO expression. PC with typical patterns of growth showed higher levels of cytoplasmic TSPO than follicular thyroid lesions. Cytoplasmic staining with anti-TSPO antibodies in PC is consistent with previously reported data showing mitochondrial TSPO localization. A recent study has demonstrated that TSPO is targeted to the outer mitochondria membrane through its interactions with cytosolic chaperones (HSC70 and HSP90) and Metaxin 1 (Mtx1) (Rone et al. 2009). An association between HSP90 expression and the PC oncogene RET/PTC1 has been shown previously (Marsee et al. 2004), suggesting that mechanisms controlling mitochondrial TSPO localization could be specifically activated in PC. Previous studies also suggested the role of cell-type-specific mechanisms controlling TSPO post-transcriptional and post-translational modification, polymerization, and/or proteasomal degradation. It has been shown that free radicals are implicated in TSPO polymerization and localization (Veenman et al. 2008). Additional studies are needed, however, to determine the exact molecular mechanisms underlying post-transcriptional TSPO modification, intracellular localization, and the biological significance of cytoplasmic TSPO expression in thyroid cancer cells.

Studies on molecular mechanisms controlling TSPO expression demonstrated that the TSPO gene promoter contains several binding sites for transcription factors, including activator protein 1 (AP1), specificity protein $1 / 3$ (SP1/SP3), signal transducer and activation of transcription (STAT3), and c-Jun (Batarseh et al. 2010). Indeed, recent data showed that MAPK/ERK signaling is implicated in the regulation of TSPO expression through c-Jun and STAT3 in Leydig cells (Batarseh et al. 2010). Of interest, constitutive activation of this same MAPK/ERK signaling due to $R A S$, 
$R E T / P T C$, or $B R A F$ mutations is common in papillary thyroid cancers (Rone et al. 2009). To determine the potential role of thyroid oncogenes in the regulation of TSPO expression, we performed in vitro experiments using pharmacological inhibitors of MAPK/ERK and PI3K/AKT signaling pathways. This analysis did not reveal any significant associations between thyroid oncogene mutations and the level of TSPO expression in thyroid cancer cells.

A recent publication demonstrated the role of epigenetic mechanisms in the regulation of TSPO expression in breast cancer cell lines (Batarseh et al. 2012). In our study, we examined the effects of the well-characterized histone deacetylase inhibitor valproic acid on TSPO expression in thyroid cancer cells. We found that treatment with valproic acid was associated with induction of TSPO expression in thyroid cancer cells with intrinsically low levels of TSPO. These data corroborate previously published results in breast cancer cell lines and confirm the role of epigenetic mechanisms in the regulation of TSPO expression.

To determine the functional role of TSPO in thyroid cancer, we performed in vitro experiments using well-characterized thyroid cancer cell lines. TSPO silencing had no effects on thyroid cancer cell growth or migration but significantly affected the thyroid cancer cell response to apoptotic stimuli. For induction of apoptosis, we used an $\mathrm{H}_{2} \mathrm{O}_{2}$-inducible oxidative stress model based on the fact that radiation, which is a mainstay of therapy for thyroid cancer, induces oxidative stress. Oxidative stress was associated with mitochondrial membrane depolarization in thyroid cancer cells in dose- and cell-type-specific manner. Inhibition of TSPO attenuated $\mathrm{H}_{2} \mathrm{O}_{2}$-inducible mitochondrial membrane depolarization in thyroid cancer cells. The TSPO antagonist PK11195 consistently prevented mitochondrial membrane depolarization in oxidative stress conditions. These findings corroborated previously published data demonstrating that inhibition of TSPO expression, as well as treatment with PK11195, protected glioblastoma cells against the pro-apoptotic effects of chemotherapeutic compounds and a hypoxia-mimicking agent (Kugler et al. 2008, Zeno et al. 2009).

Our in vitro data showed low levels of TSPO expression in cancer cells that were derived from recurrent thyroid lesions and suggested that loss of TSPO could be one of the underlying causes of cancer cell resistance to radioiodine treatment. Data from gene expression profiling (Geo Profiles), however, indicates that TSPO expression can be decreased in aggressive tumors. For example, the TSPO mRNA level was lower in recurrent colon cancer compared with tumors without relapse, and in aggressive androgen-independent prostate cancers compared with androgen-dependent tumors. In this setting, detection of TSPO expression in thyroid cancer could be used for prediction of thyroid cancer cell response to radioiodine treatment. Additional studies using a cohort of patients with well-documented responses to radioiodine treatment, as well as studies using animal models, however, will be needed before a definitive conclusion may be reached.
As treatment with valproic acid was associated with induction of TSPO, we next examined the effects of valproic acid on the response of thyroid cancer cells to oxidative stress. Our results showed that induction of TSPO by valproic acid was associated with increased sensitivity of thyroid cancer cells to oxidative stress. There is an ongoing clinical trial with valproic acid for the treatment of thyroid cancer patients that failed conventional therapy. Our results suggest that treatment with valproic acid, in addition to its known effects on induction of NIS expression and iodine uptake in thyroid cancer cells, could also increase efficiency of radiotherapy through regulation of TSPO and sensitization to oxidative stress inducible apoptosis.

Overall, we showed that during thyroid tumorigenesis, TSPO expression is associated with specific thyroid tumor phenotypes. In vitro data suggested a role for epigenetic mechanisms in the regulation of TSPO expression in thyroid cells. Implication of TSPO in the thyroid cancer cell response to oxidative stress suggested its potential role in the regulation of thyroid cancer cell response to the treatment with radioiodine and warrants further investigation.

\section{Declaration of interest}

The authors declare that there is no conflict of interest that could be perceived as prejudicing the impartiality of the research reported.

\section{Funding}

The study was supported by the American Thyroid Association Grant to J K-G and by Grant from Department of Pediatrics USUHS (C086IZ) to V V V.

\section{References}

Akech J, Sinha Roy S \& Das SK 2005 Modulation of cholinephosphotransferase activity in breast cancer cell lines by Ro5-4864, a peripheral benzodiazepine receptor agonist. Biochemical and Biophysical Research Communications 333 35-41. (doi:10.1016/j.bbrc.2005.05.071)

Batarseh A, Li J \& Papadopoulos V 2010 Protein kinase Cepsilon regulation of translocator protein $(18 \mathrm{kDa})$ Tspo gene expression is mediated through a MAPK pathway targeting STAT3 and c-Jun transcription factors. Biochemistry 49 4766-4778. (doi:10.1021/bi100020e)

Batarseh A, Barlow KD, Martinez-Arguelles DB \& Papadopoulos V 2012 Functional characterization of the human translocator protein $(18 \mathrm{kDa})$ gene promoter in human breast cancer cell lines. Biochimica et Biophysica Acta 1819 38-56. (doi:10.1016/j.bbagrm.2011.09.001)

Chelli B, Lena A, Vanacore R, Da Pozzo E, Costa B, Rossi L, Salvetti A, Scatena F, Ceruti S, Abbracchio MP et al. 2004 Peripheral benzodiazepine receptor ligands: mitochondrial transmembrane potential depolarization and apoptosis induction in rat C6 glioma cells. Biochemical Pharmacology 68 125-134. (doi:10.1016/j.bcp.2004.03.008)

Corsi L, Geminiani E \& Baraldi M 2008 Peripheral benzodiazepine receptor (PBR) new insight in cell proliferation and cell differentiation review. Current Clinical Pharmacology 3 38-45. (doi:10.2174/ 157488408783329878)

Fafalios A, Akhavan A, Parwani AV, Bies RR, McHugh KJ \& Pflug BR 2009 Translocator protein blockade reduces prostate tumor growth. Clinical Cancer Research 15 6177-6184. (doi:10.1158/1078-0432.CCR-09-0844) 
Hardwick M, Fertikh D, Culty M, Li H, Vidic B \& Papadopoulos V 1999 Peripheral-type benzodiazepine receptor (PBR) in human breast cancer: correlation of breast cancer cell aggressive phenotype with PBR expression, nuclear localization, and PBR-mediated cell proliferation and nuclear transport of cholesterol. Cancer Research 59 831-842.

Kugler W, Veenman L, Shandalov Y, Leschiner S, Spanier I, Lakomek M \& Gavish M 2008 Ligands of the mitochondrial $18 \mathrm{kDa}$ translocator protein attenuate apoptosis of human glioblastoma cells exposed to erucylphosphohomocholine. Cellular Oncology 30 435-450.

Liu Z, Hou P, Ji M, Guan H, Studeman K, Jensen K, Vasko V, El-Naggar AK \& Xing M 2008 Highly prevalent genetic alterations in receptor tyrosine kinases and phosphatidylinositol 3-kinase/akt and mitogen-activated protein kinase pathways in anaplastic and follicular thyroid cancers. Journal of Clinical Endocrinology and Metabolism 93 3106-3116. (doi:10.1210/ jc.2008-0273)

Maaser K, Hopfner M, Jansen A, Weisinger G, Gavish M, Kozikowski AP, Weizman A, Carayon P, Riecken EO, Zeitz M et al. 2001 Specific ligands of the peripheral benzodiazepine receptor induce apoptosis and cell cycle arrest in human colorectal cancer cells. British Journal of Cancer $\mathbf{8 5}$ 1771-1780. (doi:10.1054/bjoc.2001.2181)

Maaser K, Grabowski P, Oezdem Y, Krahn A, Heine B, Stein H, Buhr H, Zeitz M \& Scherubl H 2005 Up-regulation of the peripheral benzodiazepine receptor during human colorectal carcinogenesis and tumor spread. Clinical Cancer Research 11 1751-1756. (doi:10.1158/10780432.CCR-04-1955)

Marsee DK, Venkateswaran A, Tao H, Vadysirisack D, Zhang Z, Vandre DD \& Jhiang SM 2004 Inhibition of heat shock protein 90, a novel RET/PTC1-associated protein, increases radioiodide accumulation in thyroid cells. Journal of Biological Chemistry 279 43990-43997. (doi:10.1074/ jbc.M407503200)

Papadopoulos V, Baraldi M, Guilarte TR, Knudsen TB, Lacapere JJ, Lindemann P, Norenberg MD, Nutt D, Weizman A, Zhang MR et al. 2006 Translocator protein $(18 \mathrm{kDa})$ : new nomenclature for the peripheral-type benzodiazepine receptor based on its structure and molecular function. Trends in Pharmacological Sciences 27 402-409. (doi:10.1016/j.tips.2006. 06.005)

Rone MB, Liu J, Blonder J, Ye X, Veenstra TD, Young JC \& Papadopoulos V 2009 Targeting and insertion of the cholesterol-binding translocator protein into the outer mitochondrial membrane. Biochemistry 48 6909-6920. (doi:10.1021/bi900854z)

Satoh T, Enokido Y, Aoshima H, Uchiyama Y \& Hatanaka H 1997 Changes in mitochondrial membrane potential during oxidative stress-induced apoptosis in PC12 cells. Journal of Neuroscience Research 50 413-420. (doi:10 1002/(SICI)1097-4547(19971101)50:3 < 413::AID-JNR7 > 3.0.CO;2-L)
Schlumberger M \& Sherman SI 2009 Clinical trials for progressive differentiated thyroid cancer: patient selection, study design, and recent advances. Thyroid 19 1393-1400. (doi:10.1089/thy.2009.1603)

Shen WT, Wong TS, Chung WY, Wong MG, Kebebew E, Duh QY \& Clark OH 2005 Valproic acid inhibits growth, induces apoptosis, and modulates apoptosis-regulatory and differentiation gene expression in human thyroid cancer cells. Surgery 138 979-984 (discussion 984-975). (doi:10.1016/j.surg.2005.09.019)

Shoukrun R, Veenman L, Shandalov Y, Leschiner S, Spanier I, Karry R, Katz Y, Weisinger G, Weizman A \& Gavish M 2008 The 18-kDa translocator protein, formerly known as the peripheral-type benzodiazepine receptor, confers proapoptotic and antineoplastic effects in a human colorectal cancer cell line. Pharmacogenetics and Genomics 18 977-988. (doi:10.1097/FPC.0b013e3283117d52)

Sutter AP, Maaser K, Gerst B, Krahn A, Zeitz M \& Scherubl H 2004 Enhancement of peripheral benzodiazepine receptor ligand-induced apoptosis and cell cycle arrest of esophageal cancer cells by simultaneous inhibition of MAPK/ERK kinase. Biochemical Pharmacology 67 1701-1710. (doi:10.1016/j.bcp.2004.01.009)

Takaya S, Hashikawa K, Turkheimer FE, Mottram N, Deprez M, Ishizu K, Kawashima H, Akiyama H, Fukuyama H, Banati RB et al. 2007 The lack of expression of the peripheral benzodiazepine receptor characterises microglial response in anaplastic astrocytomas. Journal of Neuro-Oncology 85 95-103. (doi:10.1007/s11060-007-9396-1)

Veenman L, Shandalov Y \& Gavish M 2008 VDAC activation by the $18 \mathrm{kDa}$ translocator protein (TSPO), implications for apoptosis. Journal of Bioenergetics and Biomembranes 40 199-205. (doi:10.1007/s10863-0089142-1)

Vlodavsky E \& Soustiel JF 2007 Immunohistochemical expression of peripheral benzodiazepine receptors in human astrocytomas and its correlation with grade of malignancy, proliferation, apoptosis and survival. Journal of Neuro-Oncology 81 1-7. (doi:10.1007/s11060-006-9199-9)

Zeno S, Zaaroor M, Leschiner S, Veenman L \& Gavish M 2009 CoCl(2) induces apoptosis via the $18 \mathrm{kDa}$ translocator protein in U118MG human glioblastoma cells. Biochemistry 48 4652-4661. (doi:10.1021/bi900064t)

Received in final form 17 May 2012

Accepted 29 May 2012

Made available online as an Accepted Preprint 29 May 2012 